\title{
Image features for quality analysis of thick blood smears employed in malaria diagnosis
}

Wendy Marcela Fong Amarís ( $\square$ wendymarce110@hotmail.com )

Pontificia Universidad Javeriana https://orcid.org/0000-0001-7185-9478

Carol Viviana Martinez Luna

University of Luxembourg: Universite du Luxembourg

Liliana Jazmín Cortés Cortés

Colombian National Institute of Health

Daniel Ricardo Suárez Venegas

Pontificia Universidad Javeriana Facultad de Ingenieria

Research

Keywords: Image processing, Coloration quality, Malaria diagnosis, TBS

Posted Date: April 9th, 2021

DOI: https://doi.org/10.21203/rs.3.rs-403293/v1

License: (c) (1) This work is licensed under a Creative Commons Attribution 4.0 International License. Read Full License 


\section{Abstract}

Background: The World Health Organization (WHO) provides protocols for the diagnosis of malaria. One of them is related to the staining process of blood samples to guarantee the correct parasite visualization. Ensuring the quality of the staining procedure on thick blood smears (TBS) is a dicult task, especially in rural centers, where there are factors that can a ect the smear quality (e.g. types of reagents employed, place of sample preparation, among others). This work presents an analysis of an imagebased approach to evaluate the coloration quality of the staining process of TBS used for malaria diagnosis.

Methods: According to the WHO, there are di erent coloration quality descriptors of smears. Among those, the background color is one of the best indicators of how well the staining process was conducted. An image database with 420 images (corresponding to 42 TBS samples) was created for analyzing and testing image-based algorithms to detect the quality of the coloration of TBS. Background segmentation techniques were explored (based on RGB and HSV color spaces) to separate the background and foreground (leukocytes, platelets, parasites) information. Then, di erent features (PCA, correlation, Histograms, variance) were explored as image criteria of coloration quality on the extracted background information; and evaluated according to their capability to classify images as with Good or Bad coloration quality from TBS.

Results: For background segmentation, a thresholding-based approach in the SV components of the HSV color space was selected. It provided robustness separating the background information independently of its coloration quality. On the other hand, as image criteria of coloration quality, among the 19 feature vectors explored, the best one corresponds to the 15-bins histogram of the Hue component with classi cation rates of $>97 \%$.

Conclusions: An analysis of an image-based approach to describe the coloration quality of TBS was presented. It was demonstrated that if a robust background segmentation is conducted, the histogram of the $\mathrm{H}$ component from the HSV color space is the best feature vector to discriminate the coloration quality of the smears. These results are the baseline for automating the estimation of the coloration quality, which is a key component for automating malaria diagnosis of TBS, that has not been studied before.

\section{Full Text}

This preprint is available for download as a PDF.

\section{Figures}




\section{Step 1 \\ Database Creation of images from Thick Blood Smears}

420 images from 42 samples.

- Labels: good/bad quality

Step 2

\section{Analysis of \\ Coloration Quality Criteria in Thick Blood Smears}

Step 3 Identification of Image-based Criteria of Coloration Quality

\section{Background Segmentation \\ Feature Extraction}

- Exploration of color spaces

- Exploration of color spaces

- Thresholding selection and evaluation

- Feature exploration of background segmentation

\section{Figure 1}

Methodological steps followed to investigate the coloration quality in thick blood smears used for malaria diagnosis. 

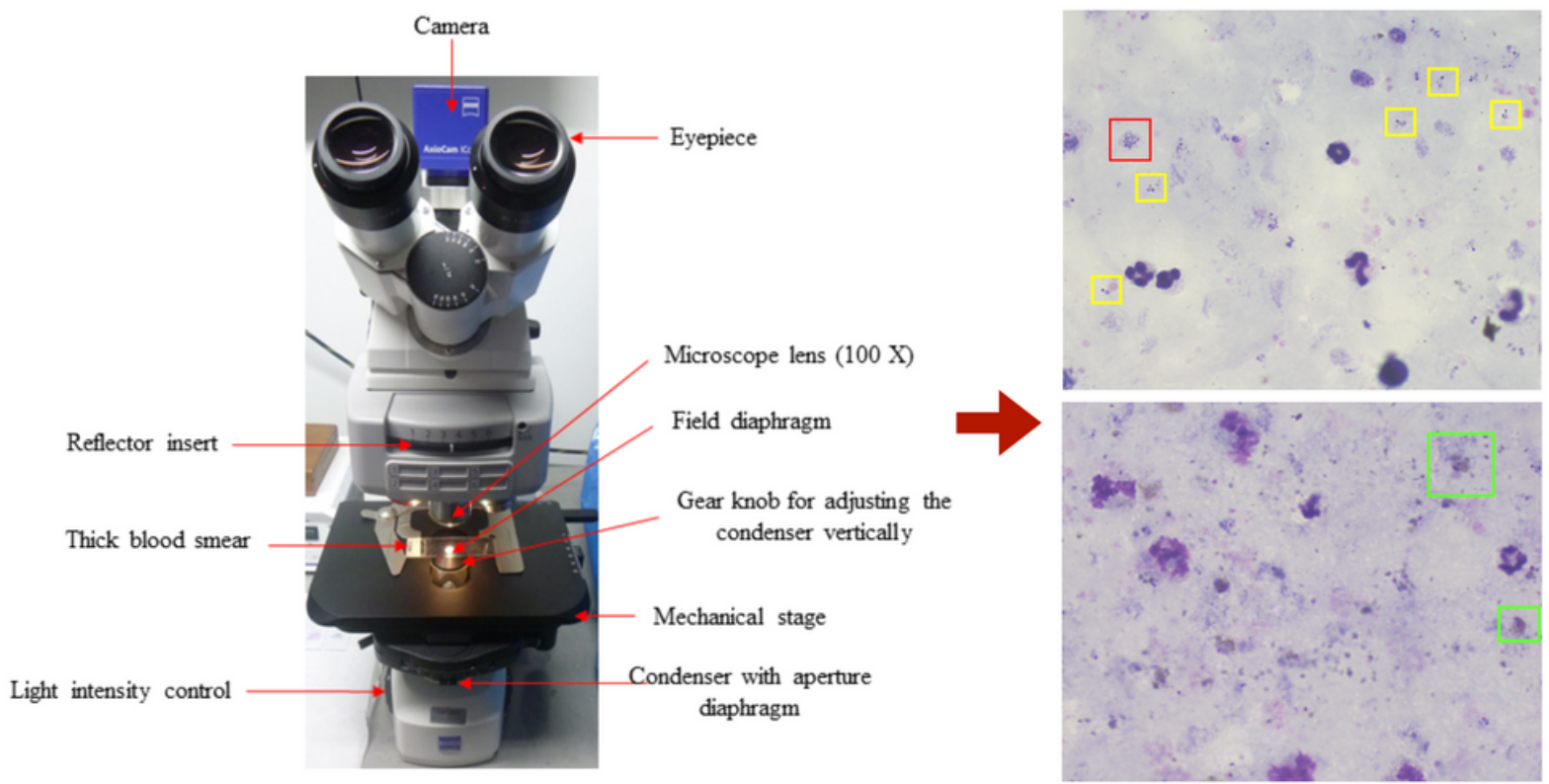

\section{P. vivax stages}

Trophozoite

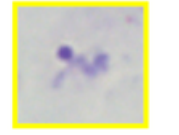

Schizont

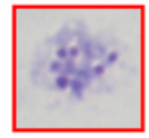

Gametocyte

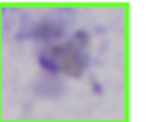

\section{Figure 2}

Optical microscope used to create the dataset of thick blood smears. It is an Axio Zeiss Scope A1, the red arrows indicate the microscope parts that were important for creating the dataset [5]. Images on the right correspond to microscopic images of thick blood smear with good coloration quality. Color boxes show the life cycle stages of the parasite P. vivax as seen in the microscope. The yellow square shows the trophozoite; red, the schizont; and green the gametocyte stage. Resolution: 1000X. Reference: Author. 


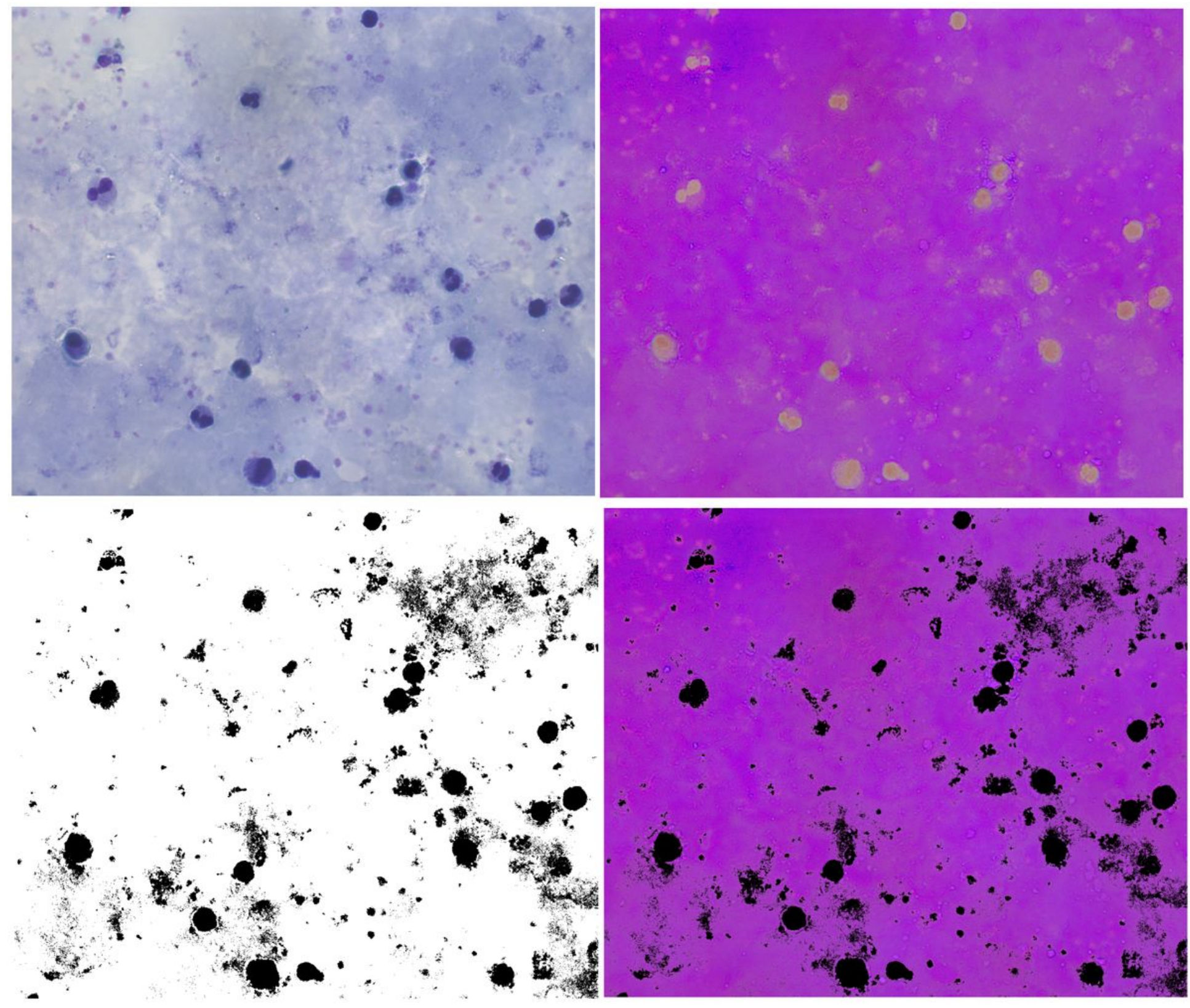

Figure 3

Background segmentation process. From left to right the gure shows the original microscopic image in RGB, the image in the HSV color space, the thresholded image (background information appears white and black), and the last image shows the background information in the HSV color space that will be used to analyze the coloration quality. 